Para enlazar con este artículo / To link to this article:

http://dx.doi.org/10.14198/fem.2017.29.01

Para citar este artículo / To cite this article:

Arenas Ramiro, Mónica. «La igualdad de oportunidades en la carrera universitaria: conciliación y corresponsabilidad como medios para conseguirla». En Marcos Jesús Iglesias Martínez e Inés Lozano Cabezas (coords.), La (in) visibilidad de las mujeres en la Educación Superior: retos y desafios en la Academia. Feminismo/s, 29 (junio 2017): 17-43, DOI: 10.14198/fem.2017.29.01

\title{
LA IGUALDAD DE OPORTUNIDADES EN LA CARRERA UNIVERSITARIA: CONCILIACIÓN Y CORRESPONSABILIDAD COMO MEDIOS PARA CONSEGUIRLA
}

\author{
EQUAL OPPORTUNITIES IN THE UNIVERSITY CAREER: \\ BALANCE AND CORRESPONSIBILITY AS A MEANS TO \\ ACHIEVE IT
}

\author{
Mónica ARENAS RAMIRO \\ Universidad de Alcalá \\ monica.arenas@uah.es \\ orcid.org/0000-0002-9329-2246
}

\section{Resumen}

A pesar de la elevada presencia femenina en el mundo universitario, y de la existencia de normas que garantizan formalmente la igualdad entre mujeres y hombres, la realidad sigue demostrando que las mujeres no gozan de las mismas oportunidades que los hombres para participar en la vida universitaria y, mucho menos, para hacer carrera académica. La perpetuación de estereotipos de género, así como la falta de medidas efectivas de conciliación y corresponsabilidad en las tareas domésticas y de cuidados provocan que las mujeres no tengan las mismas oportunidades que los hombres para su desarrollo personal y profesional. El necesario reconocimiento constitucional de las mujeres como sujetos de derechos y de las medidas de conciliación y de corresponsabilidad como herramientas antidiscriminatorias, unido a una verdadera implicación de la institución académica y de sus integrantes, permitirá, con las mismas reglas de juego, tener las mismas oportunidades y hacer visibles a las mujeres en un mundo diseñado por y para los hombres, contribuyendo a diseñar un modelo de Universidad y de carrera académica más justo e igualitario.

Palabras clave: conciliación vida familiar y laboral, corresponsabildad, género, igualdad de oportunidades, Universidad. 


\begin{abstract}
Despite the high female presence in the University, and the existence of norms that formally guarantee equality between women and men, reality continues to show that women do not enjoy the same opportunities as men to participate in university life. And, much less, to make an academic career. The perpetuation of gender stereotypes, as well as the lack of effective measures of conciliation and co-responsibility in domestic tasks and care, mean that women do not have the same opportunities as men for personal and professional development. The necessary constitutional recognition of women as subjects of rights and measures of conciliation and co-responsibility as anti-discrimination tools, coupled with a true involvement of the academic institution and its members, will allow, with the same rules of the game, to have the same opportunities and make women visible in a world designed by and for men, contributing to the design of a model of university and academic career more just and equal.
\end{abstract}

Keywords: work-life balance, co-responsibility, gender, equal opportunities, university. 


\section{INTRODUCCIÓN}

Son numerosas las normas, a nivel internacional, comunitario y nacional, que evidencian el enorme avance que en materia de igualdad se ha producido en nuestras sociedades en el último siglo. Pero nuestro día a día nos demuestra que, aunque formalmente parezca que todos tenemos las mismas oportunidades, algo debe estar fallando porque todavía persisten importantes desigualdades entre hombres y mujeres, porque esa igualdad formal no tiene su reflejo en una «igualdad de resultados» (Figueruelo Burrieza 194-195), y un claro ejemplo lo encontramos en la Universidad.

Para entender y solucionar el problema debemos analizar la situación con perspectiva de género, teniendo en cuenta que la mayoría de las diferencias entre sexos parten, en realidad, de estereotipos sociales, esto es, de características construidas socialmente (Carmona Cuenca 74; Tomás Morales 17-32). Sólo de esta forma podremos concebir el verdadero efecto discriminador que produce su mantenimiento, alejándonos de conseguir una igualdad real y efectiva entre mujeres y hombres.

En este orden de ideas, las desigualdades entre mujeres y hombres se han hecho más perceptibles desde que la mujer accede al mercado laboral y hace tambalear los cimientos de la tradicional práctica por la que la mujer se ocupa de las labores domésticas y de cuidado de la familia y el hombre se encarga del trabajo y de «traer el dinero a casa». Era cuestión de tiempo que el barco hiciera aguas, pues estos cambios económico-sociales no tuvieron su repercusión en la práctica y las mujeres siguieron, y siguen, asumiendo el rol acaparador de la esfera doméstica y de los cuidados de la familia sin que se haya producido el necesario reparto de las responsabilidades en ese terreno. Esto ha perpetuado el hecho de que las oportunidades de las mujeres para formar parte de la esfera pública, del mundo laboral, se vean disminuidas, o incluso cercenadas, a causa de la doble carga y al sobreesfuerzo que las mismas deben soportar.

Por este motivo, es más que evidente que si queremos conseguir una igualdad real entre hombres y mujeres, y que las mujeres tengan las mismas oportunidades que los hombres, los cambios económicos, sociales y culturales deben ir acompañados de «transformaciones sustanciales en la escala de 
valores» (Ortiz Ceberio 74). Y a esta necesidad obedecen, entre otras medidas, los derechos de conciliación de la vida familiar y laboral (Aguilera Izquierdo 70). Con estas medidas se consigue, además, una mejor calidad de vida y se evitan problemas tales como el absentismo, la disminución de la productividad, el estrés laboral, el descenso de la natalidad y las repercusiones económicas aparejadas, motivados por la incompatibilidad de mantener ambas facetas, y cuyo incumplimiento crea situaciones "contaminantes" y poco sostenibles con el entorno económico, social y demográfico» (Orive Álvarez, Asián Chaves y González Limón 522; Walton 11-21; León Llorente 250 y 257; Abbott, De Cieri 303-320; Rosende 32; Sarrió Catalá 44). No obstante, a pesar de estos beneficios económicos, no podemos caer aquí en trivializar estas medidas y quedarnos en su faceta laboral como medidas de promoción del empleo, destinadas a que se trabaje más y mejor y a que las mujeres puedan compatibilizar trabajo y familia (Orive Álvarez, Asián Chaves y González Limón 523-524; Macinnes 35-71).

Para que estas medidas funcionen debe romperse también la actual tendencia (fruto de los citados estereotipos) de que sean las mujeres las que, en gran medida, recurran a los derechos de conciliación ${ }^{1}$. De ahí las necesarias medidas de corresponsabilidad que deben acompañarles, pues sólo si hay un equilibrio a la hora de asumir las responsabilidades personales y familiares, lograremos avanzar en la igualdad real de oportunidades entre mujeres y hombres (Carbonell Pérez y Ramos Santana 1275).

La conciliación no es "cosa de mujeres». La solución a los problemas de igualdad de oportunidades no pasa por conciliar la vida laboral y familiar de las mujeres, perpetuando los tradicionales roles y estereotipos de género de distribución de tareas fuera y dentro del hogar (Lousada Arochena 8). La idea es conseguir la corresponsabilidad en dichas tareas de la esfera privada y que tanto hombres como mujeres tengan las mismas oportunidades de acceder y prosperar en la esfera pública, sin que la mochila de uno sea más pesada que la del otro.

En este punto damos incluso un paso más y añadimos que la conciliación no debería ser sólo cuestión de hombres y mujeres por igual, sino que la magnitud constitucional del tema obligaría a que fuera "cosa de los poderes públicos». Existe una responsabilidad política y social que los poderes públicos deben asumir, sin descargarla en los ciudadanos y, más concretamente, en las mujeres, a las que por los estereotipos de género, el tomar la decisión de elegir entre trabajo y familia, les afectará en mayor medida (Ortiz Ceberio

1. Así lo ha reconocido el Pacto Europeo por la Igualdad de Género (2011-2020) [Conclusiones del Consejo, de 7 de marzo de 2011, sobre el Pacto Europeo por la Igualdad de Género (DOUE C 155, de 25.05.2011)]. 
75-76 y 78; Belking 1-11)², y las acabará convirtiendo en el auténtico soporte del Estado social.

Así las cosas, la Universidad no escapa a estas cuestiones. Desde que a finales del siglo XIX, en 1872, se matriculara con un permiso especial (en la Facultad de Medicina de la Universidad de Barcelona) la primera mujer española, María Elena Maseras, y, en 1916, otra mujer, Emilia Pardo Bazán, consiguiera la primera cátedra (en la Universidad Central de Madrid), el número de mujeres en la Universidad española ha ido creciendo, e incluso superando al número de hombres. Se ha pasado de estar marginadas a estar integradas en el mundo universitario (García Lastra 357 y 361-362; López de la Cruz 291-293) 3.

Pero esto no significa que en el ámbito universitario no exista desigualdad. Así, aunque la Universidad está mayoritariamente copada por mujeres y el número de mujeres graduadas es mayor que el de hombres, la realidad nuevamente, dos siglos después, nos muestra, por un lado, la persistencia de estereotipos de género en las aulas (Sánchez García y otros 331-354); y, por otro lado, que las mujeres no ocupan puestos relevantes en la institución y que su carrera académica no corre paralela a la de sus compañeros varones (Ion, Durán-Bellonch y Bernabeu Tamayo 126 y 130; Donoso, Figuera y Rodríguez Moreno 187-212; Elizondo Lopetegui 25-34; Ballarín Domingo, Gallego Méndez y Martínez Benlloch) ${ }^{4}$. La Universidad parece resistirse a una igualdad real en sus dominios amparada en la creencia de que es «una institución neutra» porque sus normas de organización y funcionamiento así lo proclaman y porque se basa en la exigencia del mérito y la capacidad para el acceso y promoción en la carrera académica (Ventura Franch 2008, 160-161).

2. El sacrificio que vienen realizando muchas mujeres de su carrera profesional por su vida familiar ha alcanzado cifras tan altas, que en países como Estados Unidos el fenómeno ha recibido el nombre de la «Revolución del Abandono» («Opt-Out-Revolution»).

3. Recordamos aquí la Real Orden, de 8 de marzo de 1910, del Ministerio de Instrucción Pública y Bellas Artes, que derogaba la Real Orden, de 8 de junio de 1888, por la cual la mujer tenía que pedir un permiso especial si quería matricularse oficialmente en estudios superiores; y la Real Orden, de 2 de septiembre de 1910, del Ministerio de Instrucción Pública, por la que se abrió la posibilidad a las mujeres de opositar y concursar para los mismos puestos que los hombres.

4. Podemos citar entre otros estudios oficiales que recogen esta realidad, el publicado en 2007 por el Ministerio de Educación y Ciencia, Académicas en Cifras 2007 (http://www. amit-es.org/sites/default/files/pdf/publicaciones/academicas\%20en\%20cifras.pdf); el publicado en 2010 por el Ministerio de Sanidad, Política Social e Igualdad, Igualdad de Mujeres y Hombres en las Universidades españolas (http://www.inmujer.gob.es/areasTematicas/estudios/serieEstudios/docs/igualdadUniversidades.pdf); o el publicado en 2016 por el Ministerio de Economía, Industria y Competitividad, Científicas en Cifras 2015 (http:// www.idi.mineco.gob.es/stfls/MICINN/Ministerio/FICHEROS/Informe_Cientificas_en_ Cifras_2015_con_Anexo.pdf). 
Por ello, las medidas antidiscriminatorias suelen generar ciertas resistencias, y las situaciones de discriminación perduran, especialmente, en las condiciones y promoción profesional de su profesorado (Mentado Labao, Montané y Olivé Ferrer 1155; Caruncho Luz y Díaz Amada 1155). Pero esto no sólo ocurre a nivel europeo, sino que estudios comparados evidencian que en la mayoría de los países las mujeres sufren discriminación en el terreno universitario (Lecuona Naranjo 145; López Chocero y Sanchís Vidal 964). El problema vendrá porque en este terreno, «las desigualdades proceden de elementos informales más difíciles de detectar, analizar y resolver» (Gómez Cama 10).

Pero esto no deja de ser, cuanto menos curioso, si partimos de la base de que la universidad es una institución que contribuye al desarrollo de las personas, a su formación en valores y a la creación de un pensamiento crítico. Difícilmente podrá educar en igualdad si la misma no existe en sus aulas ni entre el personal que la compone. La Universidad debe ser el espejo de la sociedad que queremos tener, pues es la encargada de formar personas, a los profesionales del futuro, y en pleno siglo XXI esto lo debe hacer defendiendo la igualdad efectiva entre mujeres y hombres, formando a personas concienciadas con esta perspectiva de género (López-Francés y Vázquez Verdera 242; Lecuona Naranjo 158) y, por este motivo, debe predicar con el ejemplo. Así, a ello van a contribuir las medidas de conciliación, acompañadas de la correspondiente corresponsabilidad con las que se logrará no sólo una mejor calidad de vida y una sociedad más competitiva, sino una sociedad más justa y comprometida, eliminando toda forma de discriminación basada en el género, posibilitando que en el terreno académico se dé una verdadera igualdad de oportunidades entre mujeres y hombres.

\section{MARCO NORMATIVO}

Sin ánimo de hacer un recorrido exhaustivo sobre la normativa de igualdad y antidiscriminatoria existente, citamos a continuación las normas que regulan la igualdad y las medidas tendentes a conseguirla haciendo especial mención a su incidencia en el terreno de la Educación Superior.

No obstante, para empezar, es de obligada referencia recordar que fue en la IV Conferencia Mundial de Mujeres, celebrada en Pekín en 1995, donde se puso de relieve la necesidad de aplicar una perspectiva de género a la hora de analizar la situación de las mujeres en la sociedad. Asimismo, en el terreno de la corresponsabilidad, como un mecanismo tendente a conseguir la igualdad efectiva entre hombres y mujeres, la Convención sobre la Eliminación de todas las formas de Discriminación contra la Mujer, aprobada por la Asamblea General de las Naciones Unidas en 1979, exigía que los Estados signatarios así 
lo reconocieran y lo hicieran efectivo (art. 5); obligación de igualdad y conciliación que también se recoge en el ámbito del Consejo de Europa en la Carta Social Europea de 1961 (arts. 2, 4, 8, 16 y 17), y que ha venido siendo puesta de manifiesto por la Organización Internacional del Trabajo (OIT) desde su Recomendación 123, de 22 de junio de 1965, sobre el Empleo de las Mujeres con responsabilidades familiares, donde se ponía de manifiesto que la necesidad de conciliar vida laboral y familiar no es una cuestión exclusiva de las mujeres, sino de la familia y de la sociedad en su conjunto (Preámbulo).

Al margen de la normativa internacional, debemos indicar aquí que ha sido a nivel comunitario, desde la Unión Europea, desde donde se han elaborado y aprobado las normas más avanzadas y completas en materia de género (Freixes Sanjuan 15-66). Tanto el Derecho originario, Tratados constitutivos y la Carta de Derechos Fundamentales de la Unión Europea (CDFUE) integrada en los mismos, así como el Derecho comunitario derivado (con especial atención a las Directivas comunitarias) han entendido la igualdad como objetivo prioritario de la Unión y un valor fundamental de la misma (arts. 2 y 3 TUE, arts. 8, 153 y 157 TFUE y Título III (arts. 20 a 26, especial referencia a arts. 21 y 23) CDFUE), y han contribuido a que los Estados no sólo incorporen en sus ordenamientos internos medidas tendentes a erradicar la discriminación por razón de género, sino a hacer compatible la vida familiar y laboral como medio para conseguir la igualdad real y efectiva entre hombres y mujeres.

A nivel nacional, nuestro texto constitucional consagra tanto la igualdad formal como la material en sus artículos 14 y 9, respectivamente. Estas exigencias han tardado en ver un desarrollo legislativo. Así, en 1999 se aprobó la Ley 39/1999, para Promover la Conciliación de la vida familiar y laboral de las personas trabajadoras (Ley de Conciliación); en 2006, con el fin de contribuir a una mejor conciliación, se aprobó la Ley 39/2006, de Promoción de la Autonomía Personal y Atención a las personas en situación de dependencia (Ley de Dependencia); o, en 2012 se aprobó una batería de medidas con la Ley 3/2012, de Medidas urgentes para la reforma del Mercado laboral, tendente a flexibilizar normas relacionadas con el ámbito laboral y las cotizaciones a la Seguridad Social. Pero las normas esenciales en este terreno, que van a establecer una exigencia de igualdad y la prohibición de discriminación por razón de género van a ser la Ley Orgánica 1/2004, de Medidas de Protección Integral contra la Violencia de Género (LOVG), y la Ley Orgánica 3/2007, de Igualdad efectiva de oportunidades entre mujeres y hombres (LOI), introduciendo esta última, una relevante perspectiva en nuestro ordenamiento jurídico: la exigencia de una necesaria transversalidad, mainstreaming, de la igualdad de género con medidas encaminadas a ello como el permiso de paternidad (art. $44 \mathrm{LOI}$ ) (Desdentado Daroca 95; Ion, Durán-Bellonch y Bernabeu Tamayo 125; Rees). 
La influencia de estas normas en el mundo universitario no se hizo esperar, y aunque la Ley Orgánica 10/1983, de Reforma Universitaria, recogía la exigencia de una igualdad formal entre hombres y mujeres en la Educación Superior, la actual Ley Orgánica 6/2001 de Universidades (LOU) -con la reforma operada por la LO 4/2007, de Modificación de la LOU- recoge el guante lanzado por la LOI y la LOVG (arts. 25 LOI y 4.7 LOVG) e incorpora entre sus objetivos una política educativa que fomente la enseñanza y la investigación sobre el significado y alcance de la igualdad entre mujeres y hombres, erradicando todo tipo de discriminación, incluyendo la violencia de género como una de las manifestaciones más odiosas de discriminación contra la mujer por el mero hecho de serlo (Ventura Franch 2008, 167-168; Martín Bardera 205-206).

Debemos señalar aquí que, si bien las citadas normas en relación con el ámbito universitario contemplan el compromiso de la institución con los valores de igualdad y dignidad de la persona, y se prevé fomentar los estudios de género a nivel formativo, docente e investigador, por otro lado no terminan de desarrollarse los mecanismos para conseguirla, centrándose únicamente en aspectos formales como el reconocimiento del derecho de los estudiantes a la igualdad de oportunidades y a recibir un trato no sexista (art. 46.2.b) y j)), así como su derecho a un acceso y a becas y ayudas en condiciones de igualdad (arts. 42.3 y 45), la presencia equilibrada de hombres y mujeres en los órganos colegiados de gobierno (art. 13) y en los equipos de investigación (art. 41.4) $)^{5}$, la contratación basada en los principios de igualdad, mérito y capacidad garantizando la igualdad de oportunidades (arts. 48.3 y 64 y $75.2,76.3$ ) y la creación de Unidades de Igualdad para desarrollar los temas relacionados con la igualdad entre mujeres y hombres (Disp. Adic. Duodécima de la LO 4/2007, de modificación de la LOU), entre los que se incluyen los conocidos «Planes de igualdad» que las Universidades deberán aprobar, dentro del marco de los Planes Estratégicos de Igualdad de Oportunidades previstos por la LOI a nivel estatal (art. 17 LOI).

Debemos concluir, centrándonos en nuestro país, que si bien es cierto que la aprobación de la LOI supuso un importante avance en materia de igualdad, no es menos cierto que el hecho de que la exigencia de igualdad -ya reconocida en nuestra Constitución como un derecho fundamental y un principio inspirador de la actuación de los poderes públicos- se haya tenido que plasmar en

5. En el terreno de la Ciencia y la Investigación, la Ley 14/2011, de la Ciencia, la Tecnología y la Innovación reconoce la perspectiva de género en la investigación científica y técnica, garantizando la igualdad efectiva, la igualdad de oportunidades, entre hombres y mujeres en este ámbito (ver, entre otros, los artículos 4, 14, 16, 25, 26, 28 y, especialmente, la Disp. Adic. Decimotercera). 
normas obedece a que en la sociedad española persiste «una resistencia social a la igualdad en el tema de mujeres y hombres» (Ventura 2007, 523). Y sucede lo mismo en el terreno universitario. Esto pone de manifiesto que, a pesar de las normas citadas, en la Universidad no se garantiza una igualdad real de oportunidades entre mujeres y hombres y que la discriminación de la mujer proviene, en gran medida, del hecho de que la mujer se encuentre diluida e invisible en el ámbito doméstico (Añón Roig 15; Martín López y Díaz Fernández 636). De ahí que tengamos que analizar el origen del problema para buscar una solución al mismo más allá de las normas.

\section{EL PROBLEMA DE FONDO: LOS ROLES SOCIALMENTE ADMITIDOS}

Es innegable que, más allá de las cuestiones formales, ya sea en el sector público o en el privado, tanto el acceso o incorporación, como la promoción y las condiciones de trabajo de las mujeres no son iguales a las de los hombres. El origen de esta desigualdad injustificada, de esta discriminación de la mujer, no es otro que la pesada carga de la tradición, de lo que se entiende que son «sus obligaciones naturales» reproductivas y de cuidado (Esquembre Valdés 43-44; Martínez Sampere 54; Jennings 183), de los roles tradicional y socialmente admitidos.

Ya ha quedado dicho que la incorporación de la mujer al mundo laboral supuso un cambio de la estructura social y económica de los países, entre ellos, España. En España, a pesar del tránsito a la democracia, y de la prosperidad económica y los cambios legislativos que fueron aparejados (Ruíz de la Cuesta Fernández y Bajo García 132-133), no se produjo ningún cambio en la mentalidad de una sociedad tradicionalmente patriarcal, en la que existían unos roles bien definidos de lo que debían hacer los hombres y de lo que les correspondía a las mujeres, que era todo lo relacionado con las labores domésticas, reproductivas y el cuidado de los miembros de la familia (Martín López y Díaz Fernández 633; Lousada Arochena 7).

La cuestión es que estos estereotipos se aprenden, graban y reproducen por los individuos «como si de una segunda piel se tratara, actuando como una profecía autocumplida» (Sarrió Catalá 46; Merton 193-210), y acaban creando «una realidad discriminadora y segregada» (Sarrió Catalá 41). Por este motivo, la transformación laboral sufrida a lo largo de este último siglo debe ir acompañada de un reposicionamiento de los roles que los individuos tienen en la sociedad, pero tanto los de los hombres como los de las mujeres (Kaufmann Hahn 29 y 32), con el fin de que la ética del cuidado no sea sólo cosa de las mujeres, las relaciones de poder no se vuelquen al mismo lado de la balanza 
siempre, y romper así los estereotipos de género generadores de una evidente discriminación.

La cuestión de los roles se asimiló de la misma manera en el mundo académico, esto es, como si fuera una cuestión «intrafamiliar y femenina» (León Llorente 248). Todo ello, además, bajo el error de partida de creer que en la Universidad no hay desigualdad, provocando «un sesgo de género que perjudica claramente a las mujeres» integrantes de la comunidad universitaria: estudiantes, personal de Administración y Servicios (PAS) y, especialmente, al personal docente e investigador (PDI) (Ventura Franch 2008, 161).

La realidad y las cifras muestran que hay un mayor número de mujeres que de hombres en las aulas y con un nivel de estudios más altos -aunque debemos señalar que con diferencias importantes por ramas de conocimiento(Mentado Labao, Montané, Olivé Ferrer 1155) ${ }^{6}$. Pero esta excelencia se queda en la base. Se produce por lo tanto, tanto una discriminación horizontal como una discriminación vertical, y así, se habla de ramas de conocimiento feminizadas y de niveles de académicos desocupados (Caruncho Luz y Díaz Amada 1147; García Lastra 363). La presencia de mujeres en cargos directivos o en las estructuras de poder de la Universidad así como en las escalas más altas de la carrera universitaria es decepcionante (Ventura Franch 2007, 527-528; Tomás Folch y Guillamón Ramos 254-255)7. Y lo mismo sucede respecto del Personal de Administración y Servicios (PAS), donde las bases y los niveles más bajos los ocupan las mujeres y los grupos más altos son ocupados por hombres. Se evidencia la existencia del manido «techo de cristal» (López Chocero y Sanchís Vidal 964), o más bien, deberíamos hablar de un «suelo pegajoso», que atrapa a las mujeres impidiéndolas o dificultándolas acceder a puestos superiores (Tomás Folch y Guillamón Ramos 257; Heward). Esto es una muestra de que algo falla en el mundo académico. Y la respuesta no puede esconderse bajo el argumento de la tardía incorporación de la mujer al mercado laboral o al mundo universitario. La respuesta la encontramos nuevamente en la realidad dormida, en el ámbito privado, esto es, en las labores de cuidado y reproducción, en el número de hijos y el tiempo de dedicación a los mismos y al resto de

6. Para comprobar datos numéricos y cifras, remitimos al Plan Estratégico de Igualdad de Oportunidades (PEIO) 2014-2016, editado por el Instituto de la Mujer y el Ministerio de Sanidad, Servicios Sociales e Igualdad, pp. 37-41.

7. En el curso 2015/2016, el número de catedráticas era de 2.053 frente a los 7.779 catedráticos, tan sólo un $20,88 \%$. Y si atendemos a la máxima representación de la institución universitaria, a 2017 sólo existían 11 Rectoras frente a 73 Rectores. Cifras extraídas del Instituto de la Mujer, de las Estadísticas sobre Educación, Profesorado. 
la familia y las labores domésticas ${ }^{8}$. Esto unido, por un lado, a los estereotipos de género, donde la mujer «debe» tener una conducta cooperativa, marcada por la ausencia de ambición de poder, y, por otro lado, al hecho de que para las mismas se produce un conflicto moral y un estrés continuo por sentir no estar al $100 \%$ entregadas a la familia y a su formación y productividad (Alonso Sanz 227; Jiménez Rodrigo y Márquez Lepe 5), pueden ser la respuesta a la ausencia de mujeres en los cargos directivos y representativos en la Universidad (Tomás Folch y Guillamón Ramos 268). Así pues, son estos roles los que mantienen la «segregación en la elección de los estudios, por las implicaciones que tiene en relación a la división sexual del trabajo y el estatus de las carreras feminizadas» (Caruncho Luz y Díaz Amada 1146; Ion, Durán-Bellonch y Bernabeu Tamayo 126; Lunn 77-90; Leathwood y Read). De esta forma, se priva a la sociedad de un modelo femenino de referencia «y de éxito» vinculado al mundo académico (García Lastra 364).

Así las cosas, con el fin de contribuir a una igualdad efectiva entre hombres y mujeres, y propiciar que las mujeres tengan las mismas oportunidades que los hombres, las medidas de conciliación y la exigencia de corresponsabilidad parecen ser las medidas más apropiadas para conciliar vida familiar y laboral y asumir las tareas familiares y domésticas de manera corresponsable y democrática por los miembros de la familia.

Pero debemos hacer especial hincapié en que para que estas medidas no sigan perpetuando los citados estereotipos de género y se queden en papel mojado, las mimas no deben concebirse como una cuestión femenina, ni quedarse y solucionarse en la esfera privada de los individuos. Los poderes públicos tienen la misión de actuar en este terreno. La solución contraria conllevaría, como ha quedado dicho, a la perpetuación de los roles de género y al hecho de que sean las mujeres las que sientan la presión y el estrés de tener que elegir entre una vida familiar y una vida laboral, o la forma de conciliar ambas con el malabarismo de llevar a cabo una doble jornada de trabajo (Aguilera Izquierdo 69; Ruíz de la Cuesta Fernández y Bajo García 133; Carbonell Pérez y Ramos Santana 1275; Presser 46-76; Tobío; Méda). Hay que evitar, como ha reconocido nuestro Tribunal Constitucional (SSTC 185/2005 y 233/2007), que el uso de los derechos de conciliación dificulte la integración laboral de la mujer y la destierren profesionalmente. Si el Estado mantiene los roles tradicionalmente asumidos, lo que hace, además de permitir una discriminación por razón de

8. Los datos demuestran que de las personas entre 25 a 54 años que tienen hijos, mientras la tasa de empleo en los hombres es del 78\%, en las mujeres es del $57,8 \%$. Datos extraídos de las Estadísticas del Instituto de la Mujer, en colaboración con el Instituto Nacional de Estadística sobre Hombres y Mujeres en España 2014. 
género, es convertir a las mujeres en «supermujeres» que cargan con la obligación que a él le corresponde de soportar los costes del Estado social (Añón Roig 16 y 18; Moreno Fernández 45; Escudero Rodríguez 127).

\section{LA NECESIDAD DEL CAMBIO: LA CONCILIACIÓN Y LA CORRESPONSABILIDAD COMO RESPUESTA}

Las medidas de conciliación y, por ende, la exigencia de corresponsabilidad que las mismas fomentan (León Llorente 256), se enmarcan dentro del objetivo de promover y fomentar la igualdad de oportunidades entre mujeres y hombres. No podemos olvidar que «estableciendo relaciones igualitarias de poder se contribuye a una democratización de la sociedad y de la vida doméstica» (Orive Álvarez, Asián Chaves y González Limón 525). Si no se produce ese reparto igualitario de tareas domésticas se hace difícil conciliar vida personal y profesional, lo que, de perpetuarse los tradicionales estereotipos de género, acaba produciendo una situación discriminatoria para la mujer.

Con la batería de medidas de conciliación previstas en la LOI -desde los permisos retribuidos (por maternidad y por paternidad, o por lactancia, por ejemplo) a los permisos no retribuidos (como la reducción de jornada o las excedencias), entre otras-, se pretende romper con las desigualdades existentes en la forma de asumir responsabilidades familiares. El problema respecto de estas medidas se plantea, entre otras cuestiones, porque las mismas -que suelen conllevar una reducción del salario- van unidas, por regla general, a un momento en el que en la esfera privada se produce un aumento de los gastos, lo que dificulta aún más la capacidad de decisión del sujeto que las solicita (Ruíz de la Cuesta Fernández y Bajo García 136), lo que a día de hoy se viene haciendo de forma asimétrica y perjudicando claramente a las mujeres (Añón Roig 25-26).

Por este motivo, en el terreno de la igualdad no basta con proclamar y reconocer su existencia -lo que hemos visto que se produce-, sino que es necesario hacerla efectiva. Para ello hay que asumir, también, que las propuestas de mejora se van a encontrar con la dificultad de superar las tradicionales estructuras sociales, económicas y laborales existentes (Figueruelo Burrieza 195). Creemos que el punto de partida de dichas propuestas, tomando como base la relevancia constitucional del tema y la erradicación de cualquier tipo de discriminación (Reche Tello), debe estar enfocado en la gestión del tiempo. Si nos fijamos en las diferencias existentes entre hombres y mujeres, por los roles que tienen asumidos, el tiempo que cada sexo dedica a tareas familiares, domésticas y profesionales no es el mismo, y es la gestión del mismo, a través de medidas de conciliación y corresponsabilidad, lo que conllevará que «al 
final del día», tanto hombres como mujeres hayan tenido el mismo tiempo y las mismas oportunidades para desarrollarse personal y profesionalmente ${ }^{9}$.

Hay un aspecto en el que no entraremos por exceder el ámbito de este trabajo, pero que queremos dejar señalado por su importancia, más allá de las cuestiones de género aquí analizadas. Las medidas propuestas serán más eficaces si a la hora de gestionar el tiempo entran en juego no sólo poderes públicos, sino también el mercado. En este sentido, se habla de los llamados "planes de tiempo de las ciudades» a través de los cuales se armonizan horarios laborales, escolares y comerciales (León Llorente 255-256; Orive Álvarez, Asián Chaves y González Limón 523) ${ }^{10}$.

Así las cosas, centrándonos en las medidas de conciliación y corresponsabilidad, uno de los primeros requisitos para avanzar en la igualdad de oportunidades será reconocer la faceta constitucional de las mismas, dotándolas de la relevancia jurídica que se merecen. Con este enfoque, si trasladamos dichas medidas al ámbito universitario -donde los Planes y las Unidades de Igualdad de las universidades jugarán un importante papel-, con cambios concretos en el acceso a la carrera universitaria, en la promoción y en las condiciones en las que la misma se desarrolla, teniendo en cuenta los estereotipos de género, podremos hablar de una igualdad de oportunidades real y efectiva entre mujeres y hombres en el terreno académico.

\subsection{El enfoque constitucional}

Todas las medidas que podamos proponer en este terreno quedan vacías de contenido si no tienen el respaldo y reconocimiento que jurídicamente se merecen.

Las medidas de conciliación, y las inseparables medidas de corresponsabilidad, tienen como objetivo contribuir a la igualdad de oportunidades con el fin de que las mujeres compitan en igualdad de condiciones que los hombres, erradicando así las discriminaciones que esas diferencias ocasionan (Carmona Cuenca 73). De ahí la relevancia constitucional del tema (Reche Tello). Este es el motivo, en tanto que vinculados funcionalmente con la prohibición de discriminación, por el que los derechos de conciliación no deberían ser entendidos como una cuestión más propia de la legislación laboral, esto es, de la legislación ordinaria. Así se ha venido pronunciando nuestro Tribunal Constitucional

9. Sobre el tiempo dedicado a la familia y al hogar, mientras las mujeres dedican 4,7 horas diarias, los hombres le dedican 1.5. Datos extraídos de las Estadísticas ofrecidas por el Instituto de la Mujer, en relación con la Conciliación y los Usos del Tiempo.

10. En este sentido, la Asociación para la Comisión Nacional para la Racionalización de los Horarios Españoles (ARHOE) ha propuesto algunas medidas: impulsar el horario europeo, con objeto de armonizar comercialmente España con el resto de países. 
(Sentencias 240/1999, 203/2000, 3/2007, 233/2007 y 26/2011) que, reforzando la tutela de los derechos de conciliación, ha destacado que debemos ser conscientes de que está en juego una cuestión de relevancia constitucional en tanto que tiene como trasfondo la erradicación de cualquier forma de discriminación y no sólo la defensa de la igualdad (Fernández López 240, 243, 246 y 254; Ruíz-Rico Ruíz 19).

Pero vamos incluso un paso más allá. Con las medidas de conciliación y corresponsabilidad no se da sólo respuesta a cuestiones relacionadas con la prestación de trabajo, sino que las mismas van encaminadas, también, a proteger otro bien constitucionalmente garantizado: la familia (artículo $39 \mathrm{CE}$ ). Este bien constitucionalmente protegido debe garantizarse y desarrollarse en un régimen de corresponsabilidad, sin que sus cuidados recaigan exclusivamente sobre la mujer por el mero hecho de serlo, pues de lo contrario estaríamos incurriendo nuevamente en una causa de discriminación por razón de género.

Esta idea, enlazada con la de tener en cuenta ese ámbito doméstico, la distribución del tiempo y la posición de la mujer como sujeto de derechos, nos vuelve a plantear nuevamente la necesidad de reformular la posición de la mujer en nuestro texto constitucional (Añón Roig 20). No podemos, ni queremos cerrar este trabajo sin señalar que se hace necesario reformar el texto constitucional no sólo formalmente, sino sustancialmente, «a fin de incluir como sujetos políticos y jurídicos a las mujeres para posibilitar la configuración de las relaciones humanas en igualdad» (Esquembre Valdés 36, 49 y 50; Martínez Sampere 66; Durán Ferrer 285-287).

Sólo de esta forma será posible que la mujer participe y se relacione en la sociedad en condiciones de igualdad. Mientras, a la hora de elaborar nuestras normas y en nuestra Norma suprema, se siga ignorando la esfera privada, donde han permanecido ancladas e invisibles las mujeres, su situación desigual seguirá existiendo, pues no se les reconocerán los derechos en las mismas condiciones que a los hombres y, por lo tanto, no tendrán las mismas oportunidades para hacerse visibles como ellos (Figueruelo Burrieza 207). De ahí también la importancia de que los poderes públicos asuman el papel que les corresponde (Ruíz-Rico Ruíz 3-4).

En este sentido, las universidades, como agentes de desarrollo del pensamiento crítico y transmisoras de valores, deben contribuir a construir una sociedad más democrática e igualitaria. Esto pasa no sólo por formar personas concienciadas, sino por el hecho de que la propia institución predique con el ejemplo y reconozca el ámbito privado y sus efectos en los textos que regulan su organización y funcionamiento y que configuran y condicionan la 
carrera académica de las mujeres, para que pueda desarrollarse en igualdad de oportunidades.

\subsection{Los Planes y las Unidades de igualdad}

Los Planes de Igualdad son la herramienta estratégica de la que disponen las Unidades de Igualdad para incluir todos aquellos objetivos, estrategias y prácticas tendentes a eliminar la discriminación por razón de género (artículo 46 LOI) (López Chocero y Sanchís Vidal 978). Y como una estrategia más, los citados Planes pueden incluir las medidas de conciliación y corresponsabilidad tendentes a conseguir la igualdad real y efectiva entre mujeres y hombres.

Esta es la idea que subyace en las Unidades de Igualdad, y entre ellas, en las de las universidades, a pesar de que las mismas, así como los Planes de Igualdad fueron creados por imperativo legal (Disp. Adic. Duodécima LO 4/2007, de Modificación de la LOU). En este sentido, a día de hoy, todas las universidades cuentan con ellas ${ }^{11}$. Pero este cumplimiento formal de nada sirve si los Planes de igualdad no logran una igualdad efectiva, si no van acompañados de un compromiso institucional, así como de un correcto mecanismo de difusión, de seguimiento, y de evaluación que permita ver la efectividad de los mismos en el terreno de la igualdad de oportunidades entre hombres y mujeres en la arena universitaria (Mentado Labao, Montané y Olivé Ferrer 1163; Martínez, Lusa, Calvet, Gallego, Pons y Tura).

La mayoría de los Planes de igualdad aprobados por las universidades centran su atención en el Personal docente e investigador, y muchos de ellos contienen medidas novedosas con el fin de conciliar la vida personal y profesional, así como destinadas a facilitar la asunción de responsabilidades familiares. El problema, nuevamente, es que en la práctica, son las mujeres las que se encargarán de dar visibilidad al Plan, de recordar que la Universidad lo tiene, y de acogerse a las medidas que el Plan recoge. Por ello, es necesario que las medidas que se contengan en el mismo tengan en cuenta los ya mencionados roles de género y que vayan destinados a superarlos (Díaz Rosas y Cuevas López 2-3; Pulido Rivera 273-283). Para ello, deberán contener medidas que tengan en cuenta las desigualdades de género en el acceso, promoción y condiciones de trabajo de las universitarias, y gestionar el tiempo como variable a tener en cuenta, con el objetivo de que las mujeres tengan las mimas oportunidades que los hombres en su carrera académica.

11. Las mismas forman parte de la Red de Unidades de Igualdad de Género para la Excelencia Universitaria (RUIGEU). Pueden verse en http://www.redunidadesdeigualdad.udl.cat/ la-red/quien-forma-parte/. 


\subsection{El diseño del curriculum: un cambio en el acceso y la promoción}

A pesar de que la Universidad recoge y proclama entre sus valores principales la igualdad de oportunidades, la institución se basa en un sistema meritocrático de funcionamiento, que en último término contribuye a la exclusión de las mujeres de los puestos de poder (Ion, Durán-Bellonch y Bernabeu Tamayo 124 y 131; Alonso Sánchez 465-475), demostrando que los principios de mérito y capacidad no son suficientes para garantizar la igualdad de oportunidades en la carrera académica (Martín Bardera 246-247).

Para entender la carrera académica y la importancia del curriculum y de su diseño a lo largo de la misma, lo que acredita el mérito y capacidad de las docentes y su excelencia para concursar a las diferentes plazas que componen la carrera universitaria, debemos comenzar por recordar que la carrera universitaria es una carrera de fondo que comienza con el Doctorado que suele durar una media de unos 5 años. Señalamos este dato para hacernos una idea de que la carrera universitaria de una persona comenzará generalmente alrededor de los treinta años. Así, estaremos en presencia de una persona avanzada la treintena, sin un trabajo estable (con las consecuencias económicas que ello conlleva), en un momento de la vida en el que suele coincidir con la edad en la que formar una familia (Martín Bardera 238-239).

Así las cosas, al margen de esta cuestión vital, pero sin olvidarla, el curriculum académico se irá confeccionando, no sólo con labores docentes y de gestión, sino también con las de investigación. Y así se medirá la excelencia de los candidatos a las plazas de profesorado universitario. En un curriculum académico, entre otros méritos, además de la docencia y las labores de gestión, se tiene en cuenta la adquisición de sexenios de investigación, que se consigue a través de publicaciones de artículos en Revistas científicas o de monografías, la asistencia y participación en Congresos (nacionales e internacionales), la dirección de Tesis doctorales, la dirección de grupos de investigación o la obtención de proyectos -aunque existe un número elevado de mujeres en grupos de investigación, incluso superior al de los hombres en las ramas jurídicas, sociales y humanísticas, la cuestión reside en que no es así respecto de los puestos de dirección de los mismos (Caruncho Luz y Díaz Amada 1148)-.

La cuestión por otro lado, es que los procedimientos están formalizados. Pero el problema no es la forma, sino el fondo, la valoración de los méritos citados. Un requisito aparentemente neutro en el acceso a la carrera académica o a una plaza de profesorado universitario puede discriminar a las mujeres por no haber tenido las mismas oportunidades para alcanzarlo. Así, por ejemplo, 
nos vamos a encontrar primero con el problema de las Comisiones encargadas de acreditar las competencias y méritos de los aspirantes a las plazas de acceso a los cuerpos docentes funcionarios (Titularidad y Cátedra); y, después, con el problema de los Tribunales o Comisiones de selección que evalúan a los candidatos previamente acreditados. El problema no será la composición de los mismos, en tanto que la LOU exige la paridad en su composición (arts. 57 y 62 LOU) ni en los requisitos exigidos para demostrar la excelencia académica, sino en el hecho de si todos han podido alcanzar la misma de la misma manera, y sólo entonces se podrá hacer una valoración en igualdad de condiciones (Ventura Franch 2007, 529).

Si la valoración no se hace de forma igualitaria, la consecuencia de la desigualdad de trato es clara: las docentes e investigadoras confeccionan su curriculum con una menor promoción que la de sus compañeros, lo que conlleva que las mismas se queden en categorías más bajas (porque son las que menos requisitos exigen y los requisitos que menos tiempo demandan para ser adquiridos, aunque por contrapartida, suponen ingresos inferiores), lo que, a su vez, les priva de conseguir otros méritos académicos necesarios para escalar en la carrera académica, como por ejemplo, la dirección de proyectos de investigación o la dirección de tesis doctorales (Martín Bardera 242), lo que, a su vez, también, las hace menos relevantes en la vida universitaria y, por lo tanto, menos visibles que los hombres que sí que han ido acumulando dichos méritos porque han tenido tiempo para conseguirlos dado que su presencia en la vida doméstica y familiar no se consideraba imprescindible (Ion, DuránBellonch y Bernabeu Tamayo 131).

Como vemos, en la Universidad existen desequilibrios tanto en la etapa de formación como en la profesional y de consolidación basados en los aspectos a valorar en el curriculum académico, un curriculum cuyos criterios de evaluación «están construidos al servicio el ciclo vital y profesional masculino» (Tomás Folch y Guillamón Ramos 266-267).

Hay que tener en cuenta, por lo tanto, la gestión del tiempo en conexión con la asunción de los roles de género y la posición de poder de la mujer en el mundo académico. Lo que les falta a las mujeres para poder estar en igualdad de condiciones que sus compañeros académicos no es capacidad ni mérito, sino tiempo. Es más que evidente. Por poner sólo un ejemplo, las mujeres que deseen ser madres se van a ver obligadas a interrumpir o dejar de lado, al menos por un tiempo, su carrera profesional para dedicarse al cuidado de sus hijos. Este «parón» académico, que suele coincidir con la época de mayor productividad académica, al comienzo de la misma, influye negativamente en 
el curriculum profesional (Lecuona Naranjo 147) ${ }^{12}$. Y esta cuestión no puede obviarse.

Por este motivo, la cuestión no es sólo valorar los mismos méritos académicos para los hombres que para las mujeres, sino si las mujeres han podido conseguirlos en igualdad de condiciones y si deben existir medidas de acción positiva que contribuyan a que todos salgan del mismo punto de partida. Y la respuesta debería ser que sí. En este sentido se pronunció el Tribunal de Justicia de la Unión Europea en su Sentencia de 6 de julio de 2000, caso Göteborg, en el que se analizó el acceso de las mujeres a la Universidad en igualdad de condiciones que los hombres. Aquí, la universidad nombró catedrática a una mujer, con un curriculum inferior al del hombre con el que competía por la plaza, aplicando una medida de acción positiva conforme a la cual en caso de competir por una misma plaza, en igualdad de condiciones, se daría la plaza al sexo menos representado en esa categoría. Así, el Tribunal argumentó que la decisión se había tomado dentro de los límites de poseer una capacitación suficiente y sin vulnerar la objetividad de la contratación. Se legitimaba así la posibilidad de introducir medidas de acción positiva en los concursos de acceso a cátedra (Ventura Franch 2007, 530).

En este sentido, medidas como, por ejemplo, la iniciativa promovida por la Universidad de Barcelona de estudiar la posibilidad de dotar de ayudas económicas a las mujeres que quieran asistir a Congresos para cubrir los gastos de contratación de cuidadores, o bien, destinar ayudas económicas a facilitar la movilidad internacional de toda la familia, son un claro ejemplo a seguir. En idéntico sentido, a imitación de universidades nórdicas o anglosajonas, es realmente interesante la medida de establecer un periodo sin carga docente ni de gestión después del disfrute de un permiso por maternidad o paternidad o por el cuidado de una persona dependiente con el fin de «reparar» el periodo de interrupción científica producido (Ysàs Molinero 47). El no tener en cuenta estas cuestiones demuestra la poca sensibilidad de la Universidad, para quien con su actual proceder da a entender que la mujer durante su permiso de maternidad no ha sido productiva científicamente porque no ha querido y que con la reincorporación a su actividad deberá producir como si en su vida personal no hubiera cambiado nada que la pudiera distraer.

Por este motivo, se hace indispensable «favorecer la promoción y la excelencia erradicando posibles desequilibrios indirectos en las actividades realizadas» (Ysàs Molinero 46). Se hace necesario que los Planes de igualdad

12. Así lo puso de manifiesto la Comisión Europea en su Informe Política científica de la Unión Europea. Promover la excelencia mediante la integración de la igualdad entre géneros. Luxemburgo: Oficina de Publicaciones Oficiales de la Comunidad Europea, 2001. 
cuenten no sólo con medidas como las legalmente establecidas de tener en cuenta los periodos de crianza y de cuidado de personas dependientes (los permisos por conciliación que la ley garantiza), sino con otra serie de medidas de acción positiva que propicien una igualdad real y efectiva de oportunidades para las mujeres.

\subsection{Las condiciones de trabajo: un cambio en la permanencia}

Acabamos de comprobar cómo entre las causas de las diferencias existentes a la hora de elaborar un curriculum profesional, uno de los hándicaps más importantes es el tiempo, o más bien la falta del mismo, provocada por los estereotipos de género. Así las cosas, la ordenación del tiempo de trabajo constituye un factor esencial para conciliar la vida familiar y laboral y conseguir una real igualdad de oportunidades.

En relación con las condiciones de trabajo debemos señalar que el mundo universitario, especialmente el docente, debe superar, en este punto, otro tipo de estereotipos sociales relacionados con el tiempo de trabajo desarrollado para y en la institución. El profesorado universitario debe superar la errónea percepción que se tiene de su «flexibilidad horaria» (Ysàs Molinero 46), pues en el mundo académico en la práctica dicha flexibilidad significa un abierto 24/7 para trabajar, especialmente en el terreno de la investigación. Incluso más allá del erróneo estereotipo social sobre la labor universitaria, podríamos decir que es la propia Universidad, la que fomenta el «mito del académico como una persona "casada" con la ciencia», alejado del mundanal ruido, haciendo parecer incluso impensable, su compatibilidad con cualquier tipo de vida personal y familiar (Ortíz Ceberio 79).

Partimos, en este sentido, del hecho de que nuestras universidades responden a un modelo de organización muy masculinizado, patriarcal o con unos roles de género tradicionales, que todavía muestran cierta resistencia-incluso inconsciente, queremos pensar-a la presencia de las mujeres en la institución y que ha diseñado su organización, el acceso y la promoción a la misma en base a unos valores y roles masculinos (Ion, Durán-Bellonch y Bernabeu Tamayo 132; Guillamón Ramos 77-112).

Todo esto acaba provocando, según el estado de las cosas, que las mujeres queden relegadas a una posición de inferioridad respecto de sus compañeros varones en tanto que, por los roles tradicionalmente asumidos, ellas sí que están casadas con su «componente humano», generándoles, como ha quedado dicho, una sensación de estrés y culpabilidad por no poder compatibilizar todas las facetas de su vida como si fueran ellas las únicas obligadas a conciliar y las únicas responsables de los cuidados domésticos y familiares. 
En este orden de cosas, partiendo de estas peculiaridades del mundo docente universitario, con el fin de conciliar vida personal y laboral, sería necesario sin que ello suponga un estrés por renunciar a alguno de dichos ámbitos o sufrir una merma en la carrera docente, diseñar medidas de conciliación y corresponsabilidad para ambos sexos relacionadas con el desarrollo y desempeño de la actividad docente e investigadora. Y aquí, es relevante tener en cuenta la gestión del tiempo y un diseño de horarios que permita dicha conciliación. En este sentido, dejando de lado la dificultad de que, a día de hoy, horarios escolares, institucionales y comerciales cambien, y ante la ausencia de los ya citados «planes de tiempo de las ciudades», descendemos al terreno universitario para poner el granito de arena en este terreno.

En este sentido, los Planes de igualdad deben recoger medidas relacionadas con el centro y las condiciones de trabajo adaptadas a las necesidades de conciliación de la vida personal y laboral. Se deberían estudiar e implementar mecanismos para que los horarios docentes, la distribución de la docencia, fuera sensible y tuviera presente las necesidades del profesorado que tiene cargas familiares. Incluso podemos decir más aún, ya que las universidades mostrarían nuevamente su sensibilidad ante este tema si, al margen de esgrimir cuestiones presupuestarias, fueran más proclives a cubrir las bajas causadas por los permisos de maternidad con el fin de evitar una situación que conocen que se produce fruto de su negativa: el hecho de que la docencia se asuma por el resto de docentes del Área, Departamento o Unidad, con el consiguiente mal ambiente laboral, la reincorporación anticipada o una elección no deseada (Ysàs Molinero 47).

Al margen de estas cuestiones, por poner algunos ejemplos, entre las medidas por las que están apostando ya algunas universidades y otras que sería necesario tener en cuenta, creemos que sería conveniente la creación de zonas de juego infantiles, la creación de escuelas infantiles garantizadas no sólo para profesores, sino también para estudiantes con hijos, la creación de cambiadores para bebés o salas de lactancia (Alonso Sanz 234); y más relativas a los horarios y su flexibilización, la existencia de unas reglas (que no recomendaciones) sobre las preferencias para escoger horarios, unido a medidas como la existencia de sistemas rotativos que limiten el número de días en que un o una docente asumen los horarios más inconvenientes, que las clases de primera hora de la mañana o de última hora de la tarde no sean asumidas por profesorado que tengan hijos menores o personas a su cargo, o el hecho de prohibir convocar y realizar reuniones en días u horas conflictivas (Ysàs Molinero 46-47). Estas medidas, entre muchas otras, pueden contribuir no sólo a conciliar, sino también a fomentar la corresponsabilidad. 
Por último, si a estas medidas le unimos la potencialidad del teletrabajo, haciendo uso de las nuevas tecnologías, que evitan la presencia permanente en el puesto de trabajo -o en las aulas el caso de las estudiantes, aunque esta cuestión se tendría que compatibilizar con la exigencia de evaluación continua y presencial prevista en el Plan Bolonia-, podemos vaticinar unos buenos resultados tendentes a lograr una igualdad de oportunidades entre hombres y mujeres.

\subsection{La representación: un cambio en la visibilidad}

Como venimos repitiendo a lo largo de estas páginas, a pesar de la elevada presencia femenina en la Universidad, se produce una escasa presencia no sólo en las categorías académicas más altas, sino en los órganos de dirección y representación académicos. El origen de esta ausencia se debe a los motivos anteriormente expuestos y relacionados con la categoría académica ocupada por la mujer y, por lo tanto con el curriculum académico de la misma que le ha permitido ocupar dicha plaza.

Por lo tanto, vemos cómo para ocupar estos altos puestos en la Universidad la mujer se va a encontrar tanto con barreras internas como externas y va a seguir suponiendo una minoría, a pesar de que formalmente tanto la LOI como la LOU reconocen la presencia femenina en los cargos de gestión y representación (Díaz Rosas y Cuevas López 5 y 17-18; Carrasco Macías 75-87; Tomás Folch y Guillamón Ramos 253-275; Vázquez Cupeiro 185-219).

Finalmente, dejamos aquí señalado, sin ánimo de extendernos más en nuestro estudio, que con relación a la exigencia de la presencia de mujeres en los órganos de gobierno y de representación universitaria, se reabre el tema de las cuotas y la idea de colocar a alguien en un puesto no por su valía sino por su sexo. No podemos estar más de acuerdo con el hecho de que nos resulta curioso plantear este problema en una institución como es la Universidad que funciona a base de porcentajes y cuotas de representación de los distintos miembros de la comunidad universitaria «y nadie se cuestiona a quién se va a enviar a formar parte de un determinado órgano» (Ventura Franch, 528-529).

El problema de fondo que trasciende a esta falta de representación es la falta de proyección de un modelo femenino que rompa con los estereotipos de género tradicionalmente asumidos por la institución y que sigue manteniendo invisibles a las mujeres en el ámbito doméstico. 


\section{A MODO DE CONCLUSIÓN}

Vaya por delante el hecho de que partimos del firme convencimiento de que el reposicionamiento y emancipación de la mujer se queda en papel mojado «si persiste la desigualdad privada», si no se tiene en cuenta la esfera doméstica y su repercusión en la pública, si no se reconocen situaciones de poder iguales y si no se reconoce a las mujeres como sujetos de derechos (Esquembre Valdés 47).

Más aún, el problema no se soluciona sólo con reformular la Constitución en clave de género, sino que a esa igualdad formal -en la que se reconozca, entre otras cosas, la constitucionalidad de los derechos de conciliación (por las consecuencias discriminatorias que su no reconocimiento provoca)-, la tenemos que acompañar de las adecuadas medidas de corresponsabilidad, rompiendo los tradicionales estereotipos de género y superando la actual «crisis de los cuidados», demostrando que las responsabilidades familiares no son sólo «cosa de mujeres» y que el Estado debe asumir también su papel (Añón Roig 21; Moreno Fernández 41-56).

La Universidad como transmisora de valores debe contribuir a la ruptura de la estructura androcéntrica en la que se asienta, teniendo en cuenta la esfera privada y la gestión del tiempo, permitiendo que las mujeres accedan a las aulas, trabajen en la institución y progresen haciendo carrera académica, y sean visibles en la misma en igualdad de condiciones y con las mismas oportunidades que los hombres. Por ello, a través de sus Unidades y Planes de Igualdad, la Universidad debe apostar por implementar medidas más equilibradas y visibles, y sobre todo que respondan a la realidad y tengan en cuenta la esfera doméstica, enfatizando la corresponsabilidad (Carbonell Pérez y Ramos Santana 1295-1296). La Universidad debe contribuir a que las mujeres tengan las mismas oportunidades que los hombres a la hora de ser evaluadas, valoradas, reconocidas y situadas no sólo en la institución, sino en la sociedad en su conjunto. Todo depende de la sociedad que queramos construir, de la calidad de vida que queramos tener y de lo justos y democráticos que queramos ser, y ello no se puede hacer obviando a una parte de la población.

\section{REFERENCIAS BIBLIOGRÁFICAS}

Abbott, Jacqui y Helen De Cieri. «Influences on the provision of Work-life benefits: Management and employee perspectives». Journal of Management and Organization 14.3 (2008): 303-320.

Aguilera Izquierdo, Raquel. «Los derechos de conciliación de la vida personal, familiar y laboral en la Ley Orgánica para la Igualdad efectiva de mujeres y hombres». Revista del Ministerio de Trabajo y Asuntos Sociales 2 extra (2007): 69-119. 
Alonso Sanz, Amparo. «Conciliación de la vida estudiantil, familiar y laboral de una madre universitaria». Revista de Antropología Experimental 16 (2016): 223-236.

Alonso Sánchez, María José. «Las académicas. Profesorado universitario y género». Revista de Educación 328 (2002): 465-475.

Añón Roig, María José. « ¿Una legislación para transformar la realidad social? A propósito de la Ley para la Igualdad Efectiva de Mujeres y Hombres». Medidas de conciliación de la vida personal, laboral y familiar. Coord. María Belén Cardona Rubert. Albacete: Bomarzo, 2009, 11-38.

Ballarin Domingo, Pilar y M. Teresa Gallego Méndez e Isabel Martínez Benlloch. Libro Blanco de Los Estudios de las Mujeres en las Universidades españolas 197591. Madrid: Ministerio de Asuntos Sociales / Instituto de la Mujer, 1995.

Belking, Lisa. «The Opt-Out Revolution». New York Times Octubre, 26 (2003): $1-11$.

Carbonell Pérez, Amparo y Genoveva Ramos Santana. «La percepción de hombres y mujeres respecto a la conciliación vida laboral, personal y familiar: el caso de la Universidad de Valencia». I+G. Aportaciones a la Investigación sobre Mujeres y Género (2014): 1274-1296.

Carmona Cuenca, Encarna. «Lección 5. Igualdad de género y Derecho antidiscriminatorio. La Constitución Española de 1978». Diversidad de género e igualdad de derechos. Coord. Encarnación Carmona Cuenca. Valencia: Tirant lo Blanch, 2012, 67-79.

Carrasco Macías, María José. «Participación y poder de la mujer en las organizaciones educativas». XXI Revista de Educación, 6 (2004): 75-87.

Caruncho Luz, Varela, y Traba Díaz Amada. «Diagnóstico de la igualdad en la Universidad. Un caso práctico». Actas II Congreso Universitario Nacional Investigación y Género, 2010: 1139-1163.

Desdentado Daroca, Eva. «Lección 7. La Ley Orgánica 3/2007, de 22 de marzo, para la igualdad efectiva de mujeres y hombres (I)» Diversidad de género e igualdad de derechos. Coord. Encarnación Carmona Cuenca. Valencia: Tirant lo Blanch, 2012, 89-102.

Díaz Rosas, Francisco y Mercedes Cuevas López. «Género y liderazgo en la universidad española». Archivos Analíticos de Políticas Educativas 23(106) (2015): $1-26$.

Donoso, Trinidad, Pilar Figuera y María Luisa Rodríguez Moreno. «Barreras de género en el desarrollo profesional de la mujer universitaria». Revista de educación 355 (2011): 187-212.

Durán Febrer, María. «La constitucionalización de los derechos de género». Género, Constitución y Estatutos de Autonomía. Coords. Teresa Freixes Sanjuán, y Julia Sevilla Merino. Madrid: Instituto Nacional de Administración Pública, 2005.

Feminismo/s 29, junio 2017, pp. 17-43 
Elizondo Lopetegui, Arantxa. «La presencia de mujeres y hombres en las universidades españolas». Aequalitas: Revista jurídica de igualdad de oportunidades entre mujeres y hombres 21 (2007): 25-34.

Escudero Rodríguez, Ricardo. «Lección 9. Las políticas de igualdad de género en el ámbito laboral». Diversidad de género e igualdad de derechos. Coord. Encarnación Carmona Cuenca. Valencia: Tirant lo Blanch, 2012, 113-130.

Esquembre Valdés, Mar. «Género y Ciudadanía, Mujeres y Constitución». Feminismo/s 8 (2006): 35-51.

Fernández López, María Fernanda. «Conciliación de la vida laboral y familiar: una visión constitucional». Medidas de conciliación de la vida personal, laboral y familiar. Coord. María Belén Cardona Rubert. Albacete: Bomarzo (2009): 239-254.

Figueruelo Burrieza, Ángela. «Políticas públicas previstas para la igualdad real y efectiva». Igualdad ¿para qué? A propósito de la Ley Orgánica para la igualdad efectiva de mujeres y hombres. Eds. Ángela Figueruelo Burrieza, y M. ${ }^{a}$ Luisa Ibáñez Martínez, y Rosa M. ${ }^{a}$ Merino Hernández. Granada: Comares, 2007, 193-208.

Freixes Sanjuán, Teresa. «La igualdad de mujeres y hombres en el Derecho de la

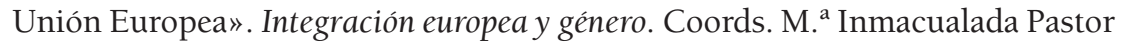
Gosálbez, y Laura Román Martín, y Ana Giménez Costa. Madrid: Tecnos, 2014, 15-66.

García Lastra, Marta. «La voz de las mujeres en la Universidad». RASE 3.3 (2010): 357-368.

Gómez Cama, M. ${ }^{\text {a }}$ del Carmen. Presencia, evolución y participación de las mujeres en la Universidad: la Universidad de Cadiz. Tesis Doctoral (inédita), 2015.

Guillamón Ramos, Cristina. «Los condicionantes de la carrera investigadora en la Universidad que encuentran las mujeres». La Universidad vista desde una perspectiva de género. Coord. Marina Tomás Folch. Barcelona: Octaedro, 2011, 77-112.

Heward, Christine. «Women and carrers in higher education. What is the problem?». Breaking Boundaries: Women in Higher Education. Eds. Louis Morley y Val Walsh. Bristol: Taylor \& Francis, 1996, 11-24.

Ion, Georgeta, María del Mar Durán-Bellonch y María Dolores Bernabeu Tamayo. «El profesorado y su percepción sobre la igualdad de género en la Universidad». Revista Complutense de Educación 24.1 (2013): 123-140.

Jennings, Ann L. «¿Público o privado? Economía institucional y feminismo». Más allá del hombre económico. Eds. Marianne A. Ferbder, y Julie A. Nelson. Madrid: Cátedra, 2004.

Jiménez Rodrigo, María Luisa y Esther Márquez Lepe. «Ir a la Universidad después de los 30: dificultades y factores facilitadores». Aula Abierta 42 (2014): 1-8. 
Kaufmann Hahn, Alicia E. «Lección 2. Psicosociología de género: los orígenes de la desigualdad familiar». Diversidad de género e igualdad de derechos. Coord. Encarnación Carmona Cuenca. Valencia: Tirant lo Blanch, 2012, 29-40.

Leathwood, Carole y Barbara Read. Gender and the changing face of higher education: a feminized future. Buckingham: Open University Press, 2008.

Lecuona Naranjo, M. ${ }^{a}$ del Pino. «Género y Universidad». Enseñanza 23 (2005): 143-160.

León Llorente, Consuelo. «Estrés laboral femenino y políticas de igualdad y flexibilidad en España». Feminismo/s 27 (2016): 243-261.

López Chocero, Sandra y Amelia Sanchís Vidal. «Mujeres en la Universidad». Actas del IV Congreso Universitario Nacional Investigación y Género. Sevilla: Universidad de Sevilla, 2012, 963-988.

López de la Cruz, Laura. «La presencia de la mujer en la Universidad española». Revista Historia de la Educación Latinoamericana 4 (2002): 291-299.

López-Francés, Inmaculada y Victoria Vázquez Verdera. «La perspectiva de género y el papel de la Universidad en el siglo XXI». TESI 15(4) (2014): 241-261.

Lousada Arochena, José Fernando. Permiso de paternidad y conciliación masculina. Albacete: Bomarzo, 2008.

Lunn, Michelle. «Women Academicians: Gender and Career Progression». Journal Pendidikan 32 (2007): 77-90.

Macinnes, John. «Diez mitos sobre la conciliación de la vida laboral y familiar». Cuadernos de Relaciones Laborales 23.1. (2005): 35-71.

Martín Bardera, Sara. Concepto de género: de las teorías feministas a las políticas públicas La universidad pública española como estudio de caso. Tesis doctoral (inédita), 2014.

Martín López, M. ${ }^{a}$ del Milagro y M. ${ }^{a}$ del Carmen Díaz Fernández. «El rol de la mujer en la Universidad: un estudio de género». Actas II Congreso Universitario Nacional «Investigación y Género», 2010, 631-647.

Martínez Sampere, Eva. «Hacia la plena ciudadanía». Género, Constitución y Estatutos de Autonomía. Coords. Teresa Freixes Sanjuán, y Julia Sevilla Merino. Madrid: Instituto Nacional de Administración Pública, 2005.

Martínez Costa, Carme, Amaia Lusa García, María Dolores Calvet Puig, Isabel Gallego Fernández, Olga Pons Peregort y Marta Tura Solvas, Guía para el diseño y la implantación de un Plan de Igualdad en las universidades. Valencia: Institut Català de les dones, 2006.

Méda, Dominique. El tiempo de las mujeres: conciliación entre vida familiar y profesional de hombres y mujeres. Madrid: Narcea, 2002.

Mentado Labao, Trinidad, Alejandrá Montané y $\mathrm{M}^{\mathrm{a}}$ Carmen Olivé Ferrer. «Espacio de género. Una política en las Universidades españolas a favor de la igualdad». Actas IV Congreso Universitario Nacional «Investigación y Género», 2012.

Merton, Robert K. «The self-fulfilling prophecy». Antioch Review 8 (1948): 193-210. 
Moreno Fernández, Louis. «Bienestar meditarráneo y «supermujeres»». Revista Española de Sociología 2 (2002): 41-56.

Orive Álvarez, M. ${ }^{a}$ José, Rosario Asián Chaves y Myriam González Limón. «Percepción de las medidas de conciliación en el personal PAS y PDI de la Universidad de Sevilla: una aproximación a su alcance». I+G. Aportaciones a la Investigación sobre Mujeres y Género (2014): 520-543.

Ortiz Ceberio, Cristina. «La (imposible) conciliación de la vida familiar y la laboral en la Universidad Norteamericana: problemas de fondo». Cuestiones de género 1 (2006): 73-86.

Presser, Harriet B. «Employment in a 24/7 economy: Challenges for the family». Fighting for time: Shifting Boundaries of Work and Social Life. Eds. C.F. Epstein y A. Kalleberg. Nueva York: Russell sage Foundation, 2004, 46-76.

Reche Tello, Nuria. La dimensión constitucional del derecho a la conciliación de la vida personal y laboral. Tesis Doctoral (inédita), 2016.

Rees, Teresa. Mainstreaming Equality in the European Union: Education, Training and Labor Market Policies. New York: Routledge, 1998.

Rosende, Magdalena. «Perspectiva comparada: medidas de conciliación de la universidad de Lausanne para el personal académico y el personal de la administración y servicios». RIDEG 01/11 (2011): 31-44.

Ruíz de la Cuesta Fernández, Sol e Irene Bajo García. «Conciliación de la vida familiar y laboral». Feminismo/s 8 (2006): 131-142.

Ruíz-Rico Ruíz, Catalina. «La problemática constitucional del derecho a conciliar la vida laboral, familiar y personal». RGDC 14 (2012): 1-19.

Sánchez García, Marifé, Magdalena Suárez Ortega, Nuria Manzano Soto, Laura Oliveros Martín-Varés, Sara Lozano Santiago, Belén Fernández D’Andrea y Beatriz Malik Liévano. «Estereotipos de género y valores sobre el trabajo entre los estudiantes españoles». Revista de Educación, 355 (2011): 331-354.

Sarrió Catalá, Maite. «Coeducación, empoderamiento y gestión de la diversidad para la conciliación de la vida privada y laboral». Medidas de conciliación de la vida personal, laboral y familiar. Coord. María Belén Cardona Rubert. Albacete: Bomarzo, 2009, 39-61.

Tobío, Constanza. Madres que trabajan: Dilemas y estrategias. Madrid: Ediciones Cátedra, 2005.

Tomás Folch, Marina y Cristina Guillamón Ramos. «Las barreras y los obstáculos en el acceso de las profesoras universitarias a los cargos de gestión académica». Revista de Educación 350 (2009): 253-275.

Tomás Morales, María Susana de. «La labor del Consejo de Europa en materia de igualdad de género». Formación y objeto del Derecho antidiscriminatorio de género: perspectiva sistemática de la igualdad desde el Derecho público. Dir. Manuela Mora Ruíz. Barcelona: Atelier, 2010, 17-32. 
Vázquez Cupeiro, Susana. «El discurso de las mujeres líderes». La segunda brecha digital. Dir. Cecilia Castaño. Madrid: Cátedra Feminismos, 2008, 185-219.

Ventura Franch, Asunción. «La integración de los estudios de las mujeres, feministas y de género en la Universidad española». Igualdad ¿para qué? A propósito de la Ley Orgánica para la igualdad efectiva de mujeres y hombres. Eds. Ángela Figueruelo Burrieza, y M. ${ }^{a}$ Luisa Ibáñez Martínez, y Rosa M. ${ }^{a}$ Merino Hernández. Granada: Comares, 2007, 523-537.

Ventura Franch, Asunción. «Normativa sobre estudios de género y Universidad». Feminismo/s 12 (2008): 155-184.

Walton, Richard E. «Quality of work life». Sloan Management Review 15.1. (1973): 11-21.

Ysàs Molinero, Helena. «Planes de igualdad y conciliación en las universidades públicas catalanas». RIDEG 01/11 (2011): 45-48. 